\title{
Depression and HIV Risk Behaviors among Female Sex Workers in Guangdong, China: A Multicenter Cross-Sectional Study
}

\author{
Hongcheng Shen, ${ }^{1}$ Huachun Zou, ${ }^{2,3}$ Shujie Huang, ${ }^{1}$ Fengying Liu, ${ }^{1}$ Peizhen Zhao, \\ Lei Chen, ${ }^{1}$ Ye Zhang, ${ }^{1}$ Xiaomin Luo, ${ }^{4}$ Weiming Tang, ${ }^{5}$ Heping Zheng, ${ }^{1}$ and Bin Yang ${ }^{1}$ \\ ${ }^{1}$ Guangdong Provincial Center for Skin Disease and STI Control, Guangzhou, Guangdong 510091, China \\ ${ }^{2}$ School of Public Health, Sun Yat-sen University, Guangzhou, Guangdong 510085, China \\ ${ }^{3}$ Kirby Institute, The University of New South Wales, Sydney, NSW 2052, Australia \\ ${ }^{4}$ Guangdong Pharmaceutical University, Guangzhou, Guangdong 511430, China \\ ${ }^{5}$ University of North Carolina Project-China, Guangzhou, Guangdong, 510091, China
}

Correspondence should be addressed to Heping Zheng; zhhpf@hotmail.com and Bin Yang; yangbin101@hotmail.com

Received 10 August 2016; Accepted 26 October 2016

Academic Editor: Abdulbari Bener

Copyright (C) 2016 Hongcheng Shen et al. This is an open access article distributed under the Creative Commons Attribution License, which permits unrestricted use, distribution, and reproduction in any medium, provided the original work is properly cited.

Background. Our study aimed to assess the burden of depression and evaluate factors associated with depression and status of HIV risk behaviors among female sex workers (FSWs) in Guangdong, China. Method. We recruited FSWs from massage parlors, saunas, restaurants, hotels, hair salons, and streets in Guangdong, China, in 2014. Information on demographic characteristics, HIV testing history, and sexual behaviors was collected using a questionnaire. A blood sample was collected to test for HIV, syphilis, and HCV. A participant was defined as being depressed if she obtained 6 points or above using the 12-item General Health Questionnaire. Results. Among the 653 participants, $41.7 \%$ were $21-30$ years old and 43.6\% married. Overall, 52.4\% were found to be depressed. FSWs who had correct syphilis related knowledge [aOR $=1.45$; 95\% CI: $1.04-2.03$ ] and had primary sex partner $(1.63,1.14-2.33)$ were more likely to be depressed. FSWs who did not use a condom during their last sex with the primary sex partner were less likely to be depressed $(0.47,0.31-0.71)$. Conclusion. Our study observed high level of depression and HIV risk behaviors among Chinese FSWs. Future interventions should integrate mental health services in comprehensive interventions to prevent depression among Chinese FSWs.

\section{Introduction}

Female sex workers (FSWs) are disproportionately affected by HIV and other sexually transmitted diseases (STDs). The pooled HIV prevalence in FSWs in 2012 was $11.8 \%$ based on data from 50 countries, including China [1]. It was estimated that there were 2-20 million FSWs in China [2-5]. FSWs are at high risk of HIV acquisition and are a bridge for the transmission of HIV/STDs to the general population [6]. A recent meta-analysis revealed that during 2000-2011 the high prevalence of STDs among FSWs became a severe public health issue in China [7].

Besides HIV/STDs, FSWs face severe stigma, sexual violence, and social discrimination [1]. As a marginalized population, they also tend to be socially isolated. FSWs are often portrayed as a symbol of social evil in China [8]. The discrimination against them became even harsher in recent years, rendering them subject to depression [9]. Existing literature on Chinese FSWs usually focused on the burden of HIV/STDs among them; however, mental health problems among this population had been neglected [10].

Globally, literature has revealed that HIV/STDs risk factors such as work-related violence [11], unwanted pregnancy [12], and condomless sex [13] were associated with mental health problems, and mental health problems in turn may increase the spread of HIV/STDs among FSWs since FSWs with high level of depression symptoms were less likely to use condom consistently or properly [8]. FSWs with mental health problem are more likely to have suicide ideation and attempt [11-14]. Limited pilot studies have indicated 
that partner violence, inconsistent condom use, and nonuse of prevention service were associated with mental health problems among FSWs in China $[15,16]$. Until now, very few studies had assessed depression among FSWs in China. Our study aimed to assess the burden of and factors associated with depression and status of HIV risk behaviors among FSWs in Guangdong, China.

\section{Methods}

2.1. Study Sample. In this multicenter, cross-sectional study, geographical mapping was used to identify the commercial sex entertainment venues. Participants in this study were recruited from massage parlors, saunas, restaurants, hotels, hair salons, and streets in three cities (Zhuhai, Nanshan District in Shenzhen, and Yingde City in Qingyuan) in Guangdong, southern China, between June and November in 2014. These three sites were chosen according to their income level: Nanshan District, a high-income area with an incidence of newly reported syphilis of 65 per 100000 person-years in 2013; Zhuhai, a medium-income area with an incidence of newly reported syphilis of 55 per 100000 person-years in 2013; and Yingde, a low-income area with an incidence of newly reported syphilis of 76 per 100000 person-years in 2013 (surveillance data not published). Convenient sampling method was used to recruit eligible participants. Outreach strategies to contact FSWs and collect data were as follows: first, trained investigators contacted the owners or managers of each establishment for their permission to conduct the survey on their premises. Second, investigators invited FSWs in participating venues to participate in our survey. Third, investigators introduced the survey to FSWs in detail and asked them to provide informed consent before completing it. Eligible participants must meet the following inclusion criteria: (1) female gender by birth, (2) aged 18 years or older, and (3) having sold sex for money or goods in the last year.

\subsection{Data Collection}

2.2.1. Demographic and Socioeconomic Measures. Trained investigators conducted face-to-face interviews using a structured questionnaire. Demographic and socioeconomic information, including age, education level, marital status, living status, monthly income, and residency, was collected from each participant. Age (in years) was measured as a continuous variable and was further categorized into different age groups $(<20 ; 21-30 ; 31-40 ; 41$ and above). Education was measured by the highest level of education obtained and categorized into primary school and below, middle school, and senior high school and above. Marital status was categorized into unmarried, married, and divorced or widowed. Living status was categorized into living alone, living with partners, and others. Monthly income was categorized into three groups (in USD): 450 and lower, 451-749, and 750 and higher. Residence was categorized into study city, another city in Guangdong province, and other provinces.

2.2.2. Behavior Characteristics. Sexual behaviors variable included experience of HIV/STDs services in the past year (including education promotion, HIV/STDs counseling and testing, and condom promotion), syphilis related knowledge, condom use during the last sex with a client, frequency of condom use in the last month with clients, condom use during the last sex with primary sex partner, frequency of condom use in the last month with primary sex partners, STD symptoms in the past year, and drug use. Participants received any kind of HIV/STD health service in the last year were considered to have experienced HIV/STD service. Participants providing correct answers to at least 6 of the 8 syphilis related knowledge questions were considered to have correct knowledge regarding syphilis transmission and prevention. Participants having any related HIV/STD symptom in last year were considered to have HIV/STD symptom. Participants having ever taken any kind of drug were considered to be drug user.

2.2.3. Depression Assessment. The depression status of the participants was assessed using the validated 12-item General Health Questionnaire (GHQ-12) [17]. The Chinese version of the questionnaire had been validated in the context of China [18]. The GHQ-12 is a screening tool which was used to screen the severity of depression experienced by an individual within the past few weeks. Each item on the scale has four answers including "better than usual," "same as usual," "less than usual," and "much less than usual." For the purpose of this study, the scoring method (0-0-1-1) was chosen. Total scores of each participant were summed up using all the items score ranging from 0 to 12 . Due to the various thresholds of the GHQ-12, the mean GHQ score for the participants was suggested as a rough indicator for the best cut-off point [19]. Therefore, based on the mean GHQ-12 score for this sample, 6 as the cut-off point was used to determine participants' level of depression.

2.2.4. Serologic Measures. Trained investigators collected $5 \mathrm{ml}$ of venous blood to test for HIV, syphilis, and HCV. HIV antibodies were screened using an enzyme-linked immune sorbent assay (ELISA; Zhuhai LiZhu Co., Ltd.). Positive samples were doubled-checked by another enzyme-linked immune sorbent assay (ELISA; Beijing Wantai Biological Pharmacy Enterprise Co., Ltd.). Samples positive by both ELISA tests were further confirmed by Western blot. Syphilis antibodies were screened using an enzyme-linked immune sorbent assay (ELISA; Zhuhai LiZhu Co., Ltd.). Positive samples were further confirmed by Toluidine Red Unheated Serum Test (TRUST; Shanghai RongSheng). Syphilis positivity was deemed as "current" when both ELISA and TRUST assays were positive. HCV antibodies were screened using an enzyme-linked immune sorbent assay (ELISA; Beijing Wantai Biological Pharmacy Enterprise Co., Ltd.). Positive samples were double-checked by another enzyme-linked immune sorbent assay (ELISA; Zhuhai LiZhu Co., Ltd.). Samples positive by both ELISA tests were defined as HCV positive.

2.3. Statistical Analysis. Data were double-entered with logic checks, using EpiData 3.0. Identified errors were corrected 
by communicating with the local chronic diseases prevention and control hospitals and rechecking the original questionnaires. Descriptive analyses were conducted to determine the distribution of the demographic factors, behaviors, HIV and syphilis prevalence, and other related information for both participants who were identified as depressed and not. Univariate logistic regression analyses with odds ratio (OR) and corresponding 95\% confidence interval (CI) were used to identify predictors of depression among the participants. To control for potential confounders, multivariate logistic regressions were further performed to determine adjusted ORs (aOR) and corresponding 95\% CIs. Variables with a $p$ value $<0.1$ was entered in the multivariate logistic regressions. Age, education level, marital status, and monthly income were also included in the multivariate logistic regression model. SAS version 9.2 (SAS Ins., Cary, NC, USA) was used for all statistical analyses.

2.4. Ethics Approval. Signed informed consent was obtained from each of the participants prior to interview and blood collection. Each of the participants was free to withdraw from this survey at any time after recruitment without any consequence. The study protocol was reviewed and approved by the Ethics Committee of the Guangdong Provincial Center for Skin Disease and STI Control in Guangzhou.

\section{Results}

3.1. Demographic Characteristics. A total of 653 participants were recruited from the three cities (120 from Shenzhen, 300 from Zhuhai, and 233 from Qingyuan). More than two-fifths $(272 / 653,41.7 \%)$ of the participants were $21-30$ years old and $43.6 \%(281 / 645)$ were married. More than half $(375 / 653,57.4 \%)$ had an education of middle school. The majority of participants had a monthly income less than USD750 (589/653, 90.2\%). Most were migrants from other provinces $(458 / 646,70.9 \%)$. More than four-fifths (415/514, $80.7 \%$ ) had received STDs related service in the last year, and approximately two-fifths $(261 / 653,40.0 \%)$ had correct syphilis related knowledge. In addition, 33.5\% (219/653) of the participants had ever experienced STDs related symptoms in the past year (Table 1).

3.2. Behavioral Characteristics. More than three-fifths (434/ $653,66.5 \%)$ of the participants self-reported that they currently had primary sex partners. Just over one-fifth (133/648, $20.5 \%$ ) of the participants did not use a condom during their last sex with a client, and 43.9\% (190/433) did not use a condom during their last sex with their primary sex partner. Among the participants, 36.6\% (239/653) engaged in condomless sex with clients in the last month, and $74.1 \%(484 / 653)$ engaged in condomless sex with primary sex partner in the last month. Only $1.1 \%(7 / 653)$ of the participants were drug users (Table 1).

3.3. HIV, Syphilis, and HCV. HIV, syphilis, and HCV were detected in $0.6 \%$ (4/653), 5.1\% (33/653), and 2.0\% (13/653) of participants. The HIV prevalence among non-depression group and depression group were $0.3 \%$ (95\% CI: $0.0-1.0 \%)$ and $0.9 \%(0.0-1.9 \%)$, respectively. Syphilis prevalence for the two groups was $6.4 \%(3.7-9.2 \%)$ and $3.8 \%(1.8-5.8 \%)$, respectively. HCV prevalence was $1.6 \%(0.2-3.0 \%)$ and $2.3 \%$ (0.7-3.9\%) among the two groups, respectively (Table 1).

3.4. Assessment of Depression. Table 2 shows the mean score for each item of GHQ-12 among the participants. Over half $(52.4 \%)$ of FSWs obtained 6 points or higher. Item 10 "been losing confidence in yourself" had the highest mean score of 0.91 , while item 7 "been able to enjoy your normal day-to-day activities" had the lowest mean score of 0.04 .

The results of univariate analysis indicated that compared to participants with a monthly income USD450 and lower, those with a monthly income USD750 and higher were more likely to be depressed $(\mathrm{OR}=1.95,1.11-3.45)$. Compared to participants from study cities, participants from other cities in Guangdong province were more likely to be depressed (OR $=2.03,1.06-3.89$ ). After adjusting for age, education, marital status, and monthly income, results from multivariate analysis indicated participants who had syphilis related knowledge $(\mathrm{aOR}=1.45,1.04,2.03)$ were more likely to be depressed compared to those who did not. The study results further indicated that FSWs who had primary sex partner were more likely to be depressed ( $\mathrm{aOR}=1.63,1.14-2.33)$. We also found that FSWs who did not use a condom during their last sex with primary sex partner $(\mathrm{aOR}=0.47,0.31-0.71)$ were less likely to be depressed. Participants tested positive for HIV or syphilis; however, they did not have statistically significantly higher rate of depression (Table 3 ).

\section{Discussion}

The current study was the first one in Guangdong to report on the severity and associated factors of depression among FSWs. The results revealed that depression and HIV risk behaviors were high among FSWs in Guangdong.

In our study, participants with syphilis related knowledge were more likely to be depressed. One potential reason for this phenomenon is that correct knowledge on syphilis may make the participants more anxious about their health when they engage in commercial sex, and, in turn, lead to the problem of depression. FSWs may expose to risk behavior or have previous or current HIV/STDs related symptoms. As a result they may be more alert to and worried about health issues, which subject them to depression. This result indicates that health care service should be strengthened to avoid depression among FSWs on a timely basis.

Our result also indicated that participants with primary sex partner were more vulnerable to depression. This finding was consistent with another study conducted in southwest China, which reported that FSWs were more likely to be victims of violence from regular partner [20]. Another study focused on FSWs in China also observed that primary violence was a constant source of stress and more detrimental effects such as alcohol intoxication and suicidal behavior [15]. In China, partners of FSWs may be financially reliant on FSWs [21]. Future intervention program should involve 
TABLE 1: Distribution of socio-demographics, sexual behavior and HIV/syphilis prevalence among female sex workers in Guangdong, China $(N=653)$.

\begin{tabular}{|c|c|c|c|c|c|c|c|c|}
\hline \multirow{2}{*}{ Item } & \multicolumn{3}{|c|}{ Non-depression $(n=311)$} & \multicolumn{3}{|c|}{ Depression $(n=342)$} & \multicolumn{2}{|c|}{ Total $(n=653)$} \\
\hline & $n$ & $\%$ & $95 \% \mathrm{CI}$ & $n$ & $\%$ & $95 \% \mathrm{CI}$ & $n$ & $\%$ \\
\hline \multicolumn{9}{|l|}{ Age (Year) } \\
\hline$\leq 20$ & 38 & 12.2 & $8.6,15.9$ & 53 & 15.5 & $11.6,19.4$ & 91 & 13.9 \\
\hline $21-30$ & 139 & 44.7 & $39.1,50.3$ & 133 & 38.9 & $33.7,44.1$ & 272 & 41.7 \\
\hline $31-40$ & 77 & 24.8 & $19.9,29.6$ & 93 & 27.2 & $22.5,31.9$ & 170 & 26.0 \\
\hline 41 and above & 57 & 18.3 & $14.0,22.7$ & 63 & 18.4 & $14.3,22.6$ & 120 & 18.4 \\
\hline \multicolumn{9}{|l|}{ Education } \\
\hline Primary school and below & 66 & 21.2 & $16.7,25.8$ & 87 & 25.4 & $20.8,30.1$ & 153 & 23.4 \\
\hline Middle school & 188 & 60.5 & $55.0,65.9$ & 187 & 54.7 & $49.4,60.0$ & 375 & 57.4 \\
\hline Senior high school and above & 57 & 18.3 & $14.0,22.7$ & 68 & 19.9 & $15.6,24.1$ & 125 & 19.1 \\
\hline \multicolumn{9}{|l|}{ Marital Status } \\
\hline Unmarried & 121 & 39.8 & $34.3,45.3$ & 152 & 44.6 & $39.3,49.9$ & 273 & 42.3 \\
\hline Married & 137 & 45.1 & $39.4,50.7$ & 144 & 42.2 & $37.0,47.5$ & 281 & 43.6 \\
\hline Others & 46 & 15.1 & $11.1,19.2$ & 45 & 13.2 & $9.6,16.8$ & 91 & 14.1 \\
\hline \multicolumn{9}{|l|}{ Living Status } \\
\hline Alone & 162 & 52.4 & $46.8,58.0$ & 165 & 48.5 & $43.2,53.9$ & 327 & 50.4 \\
\hline With partner & 72 & 23.3 & $18.6,28.0$ & 88 & 25.9 & $21.2,30.6$ & 160 & 24.7 \\
\hline Others & 75 & 24.3 & $19.5,29.1$ & 87 & 25.6 & $20.9,30.3$ & 162 & 25.0 \\
\hline \multicolumn{9}{|l|}{ Monthly income } \\
\hline$\leq 450$ & 144 & 46.3 & $40.7,51.9$ & 151 & 44.2 & $38.9,49.4$ & 295 & 45.2 \\
\hline $451-749$ & 146 & 46.9 & $41.4,52.5$ & 148 & 43.3 & $38.0,48.6$ & 294 & 45.0 \\
\hline$\geq 750$ & 21 & 6.8 & $3.9,9.6$ & 43 & 12.6 & $9.0,16.1$ & 64 & 9.8 \\
\hline \multicolumn{9}{|l|}{ Residency } \\
\hline Study city & 73 & 23.8 & $19.0,28.6$ & 61 & 18.0 & $13.9,22.2$ & 134 & 20.7 \\
\hline Another city in Guangdong province & 20 & 6.5 & $3.7,9.3$ & 34 & 10.0 & $6.8,13.2$ & 54 & 8.4 \\
\hline Another province & 214 & 69.7 & $64.5,74.9$ & 244 & 72.0 & $67.2,76.8$ & 458 & 70.9 \\
\hline \multicolumn{9}{|l|}{ STDs related Service } \\
\hline No & 53 & 21.5 & $16.3,26.6$ & 46 & 17.2 & $12.7,21.8$ & 99 & 19.3 \\
\hline Yes & 194 & 78.5 & $73.4,83.7$ & 221 & 82.8 & $78.2,87.3$ & 415 & 80.7 \\
\hline \multicolumn{9}{|l|}{ Syphilis related Knowledge } \\
\hline No & 201 & 64.6 & $59.3,70.0$ & 191 & 55.8 & $50.6,61.1$ & 392 & 60.0 \\
\hline Yes & 110 & 35.4 & $30.0,40.7$ & 151 & 44.2 & $38.9,49.4$ & 261 & 40.0 \\
\hline \multicolumn{9}{|l|}{ Condom use during the last work } \\
\hline No & 64 & 20.7 & $16.2,25.3$ & 69 & 20.4 & $16.1,24.7$ & 133 & 20.5 \\
\hline Yes & 245 & 79.3 & $74.7,83.8$ & 270 & 79.6 & $75.3,83.9$ & 515 & 79.5 \\
\hline \multicolumn{9}{|c|}{ Frequency of condom use in the last month with clients } \\
\hline Sometimes & 109 & 35.0 & $29.7,40.4$ & 130 & 38.0 & $32.8,43.2$ & 239 & 36.6 \\
\hline Always & 202 & 65.0 & $59.6,70.3$ & 212 & 62.0 & $55.8,67.2$ & 414 & 63.4 \\
\hline \multicolumn{9}{|l|}{ Primary sex partners } \\
\hline No & 86 & 27.7 & $22.7,32.7$ & 133 & 38.9 & $33.7,44.1$ & 219 & 33.5 \\
\hline Yes & 225 & 72.3 & $67.4,77.4$ & 209 & 61.1 & $55.9,66.3$ & 434 & 66.5 \\
\hline \multicolumn{9}{|c|}{ Condom use during the last sex with primary partners } \\
\hline No & 120 & 53.3 & $46.8,59.9$ & 70 & 33.7 & $27.2,40.1$ & 190 & 43.9 \\
\hline Yes & 105 & 46.7 & $40.1,53.2$ & 138 & 66.3 & $59.9,72.8$ & 243 & 56.1 \\
\hline \multicolumn{9}{|c|}{ Frequency of condom use in the last month with primary partners } \\
\hline Sometimes & 237 & 76.2 & $71.5,81.0$ & 247 & 72.2 & $67.5,77.0$ & 484 & 74.1 \\
\hline Always & 74 & 23.8 & $19.0,28.6$ & 95 & 27.8 & $23.0,32.6$ & 169 & 25.9 \\
\hline
\end{tabular}


TABle 1: Continued.

\begin{tabular}{|c|c|c|c|c|c|c|c|c|}
\hline \multirow{2}{*}{ Item } & \multicolumn{3}{|c|}{ Non-depression $(n=311)$} & \multicolumn{3}{|c|}{ Depression $(n=342)$} & \multicolumn{2}{|c|}{ Total $(n=653)$} \\
\hline & $n$ & $\%$ & $95 \% \mathrm{CI}$ & $n$ & $\%$ & $95 \% \mathrm{CI}$ & $n$ & $\%$ \\
\hline \multicolumn{9}{|c|}{ STDs Symptoms } \\
\hline No & 197 & 63.3 & $58.0,68.7$ & 237 & 69.3 & $64.4,74.2$ & 434 & 66.5 \\
\hline Yes & 114 & 36.7 & $31.3,42.0$ & 105 & 30.7 & $25.8,35.6$ & 219 & 33.5 \\
\hline \multicolumn{9}{|c|}{ Drug Use } \\
\hline No & 307 & 98.7 & $97.5,100.0$ & 339 & 99.1 & $98.1,100.0$ & 646 & 98.9 \\
\hline Yes & 4 & 1.3 & $0.0,2.6$ & 3 & 0.9 & $0.0,1.9$ & 7 & 1.1 \\
\hline \multicolumn{9}{|l|}{ HIV } \\
\hline No & 310 & 99.7 & $99.1,100.0$ & 339 & 99.1 & $98.1,100.0$ & 649 & 99.4 \\
\hline Yes & 1 & 0.3 & $0.0,1.0$ & 3 & 0.9 & $0.0,1.9$ & 4 & 0.6 \\
\hline \multicolumn{9}{|c|}{ Syphilis } \\
\hline No & 291 & 93.6 & $90.8,96.3$ & 329 & 96.2 & $94.2,98.2$ & 620 & 94.9 \\
\hline Yes & 20 & 6.4 & $3.7,9.2$ & 13 & 3.8 & $1.8,5.8$ & 33 & 5.1 \\
\hline \multicolumn{9}{|l|}{$\mathrm{HCV}$} \\
\hline No & 306 & 98.4 & $97.0,99.8$ & 334 & 97.7 & $96.1,99.3$ & 640 & 98.0 \\
\hline Yes & 5 & 1.6 & $0.2,3.0$ & 8 & 2.3 & $0.7,3.9$ & 13 & 2.0 \\
\hline
\end{tabular}

TABLE 2: Mean score of each item of GHQ-12 among female sex workers in Guangdong, China.

\begin{tabular}{|c|c|c|c|}
\hline & Non-depression $(n=311)$ & Depression $(n=342)$ & Total $(n=653)$ \\
\hline Been able to concentrate on whatever you're doing & 0.11 & 0.11 & 0.11 \\
\hline Lost much sleep over worry & 0.10 & 0.84 & 0.49 \\
\hline Felt you were playing a useful part in things & 0.09 & 0.09 & 0.09 \\
\hline Felt capable of making decisions about things & 0.06 & 0.09 & 0.08 \\
\hline Felt constantly under strain & 0.73 & 0.93 & 0.83 \\
\hline Felt you couldn't overcome your difficulties & 0.87 & 0.96 & 0.91 \\
\hline Been able to enjoy your normal day-to-day activities & 0.02 & 0.06 & 0.04 \\
\hline Been able to face up to your problems & 0.06 & 0.06 & 0.06 \\
\hline Been feeling unhappy and depressed & 0.59 & 0.90 & 0.75 \\
\hline Been losing confidence in yourself & 0.86 & 0.96 & 0.91 \\
\hline Been thinking yourself as a worthless person & 0.85 & 0.96 & 0.91 \\
\hline Been feeling reasonable happy, all things considered & 0.22 & 0.19 & 0.20 \\
\hline
\end{tabular}

primary sex partner of FSWs. We also identified that FSWs who did not use a condom during their last sex with primary sex partner were less likely to be depressed. FSWs may have barrier to use condoms with their primary sex partner as it may affect the intimacy of the relationship or as an implication of mistrust [22]. They may also see condom use as a way of distinguishing work-related sexual relationship from personal relationship [21]. FSWs tend not to use a condom during sexual intercourse with their primary sex partners. Inorder to maintain trust in their primary sexual relationship, FSWs may choose not to use a condom with their primary sex partners [23, 24]. Condom promotion should target both FSWs and their primary sex partners.

Our data indicated that an extremely high proportion (52.4\%) of the participants has the potential to develop or experience depression disorder which was higher than that reported in studies from Sydney, Australia (33.3\%, female street-based sex workers) [11], Guangxi, China (39\%, female injection drug user who are sex workers) [14], Tijuana and Ciudad Juarez, Mexico (11.8\%, female sex workers who use drugs) [21], but lower than studies from Nepal $(82.4 \%$, female sex workers) [13]. Previous studies indicated that FSWs with mental health problems were more likely to have suicide ideation and attempt [11-14] and FSWs with high level of depression symptoms were less likely to use condom consistently or properly [8] which made them at high risk of HIV/STDs infection. We searched in PubMed and the Chinese National Knowledge Infrastructure (CNKI) databases using the key words "female sex workers" and "depression" but did not find any study that reported on intervention of depression among FSWs in either China or other countries. Depression disorder has now been recognized in 
TABLE 3: Factors associated with depression among female sex workers in Guangdong, China $(N=653)$.

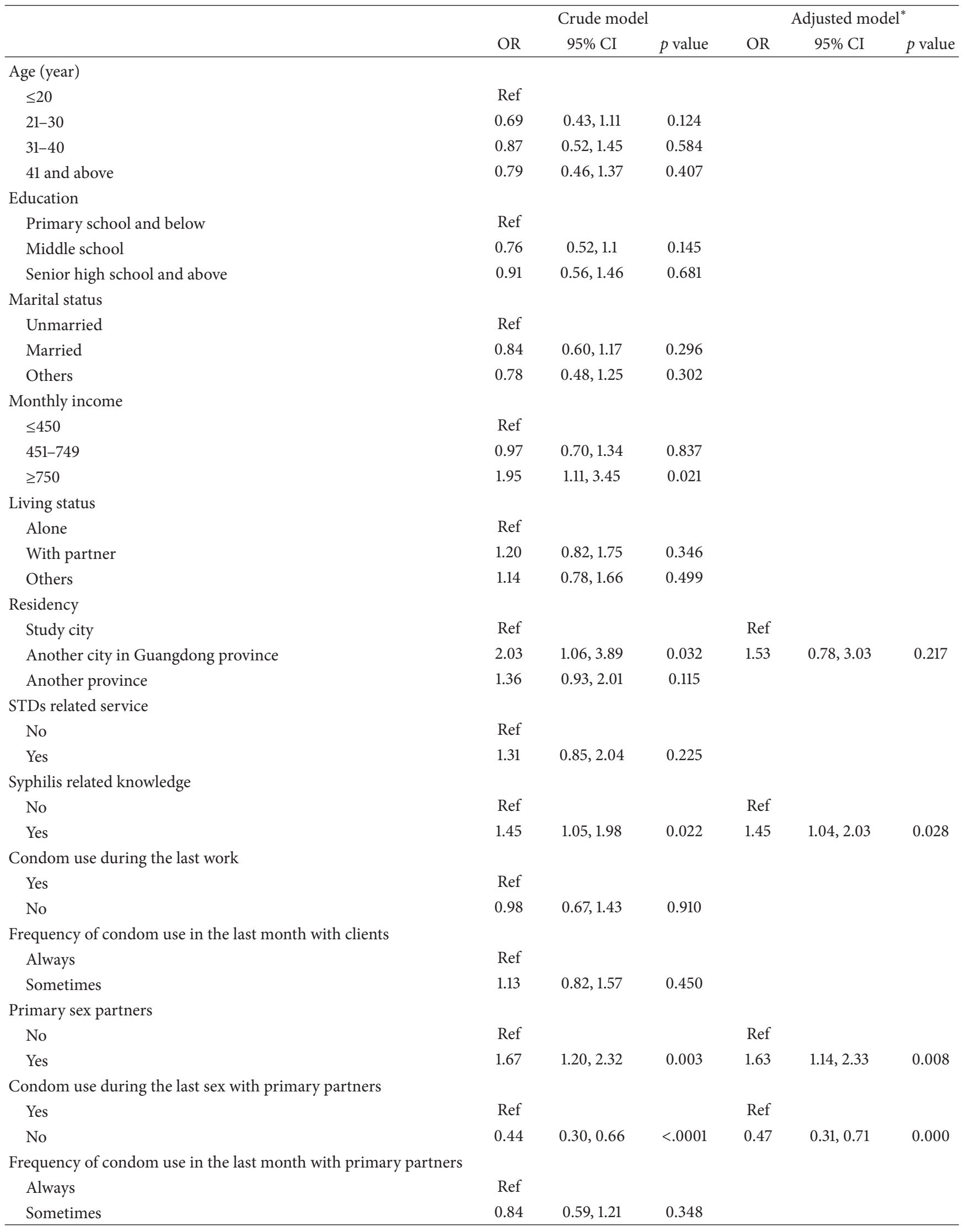


TABLe 3: Continued.

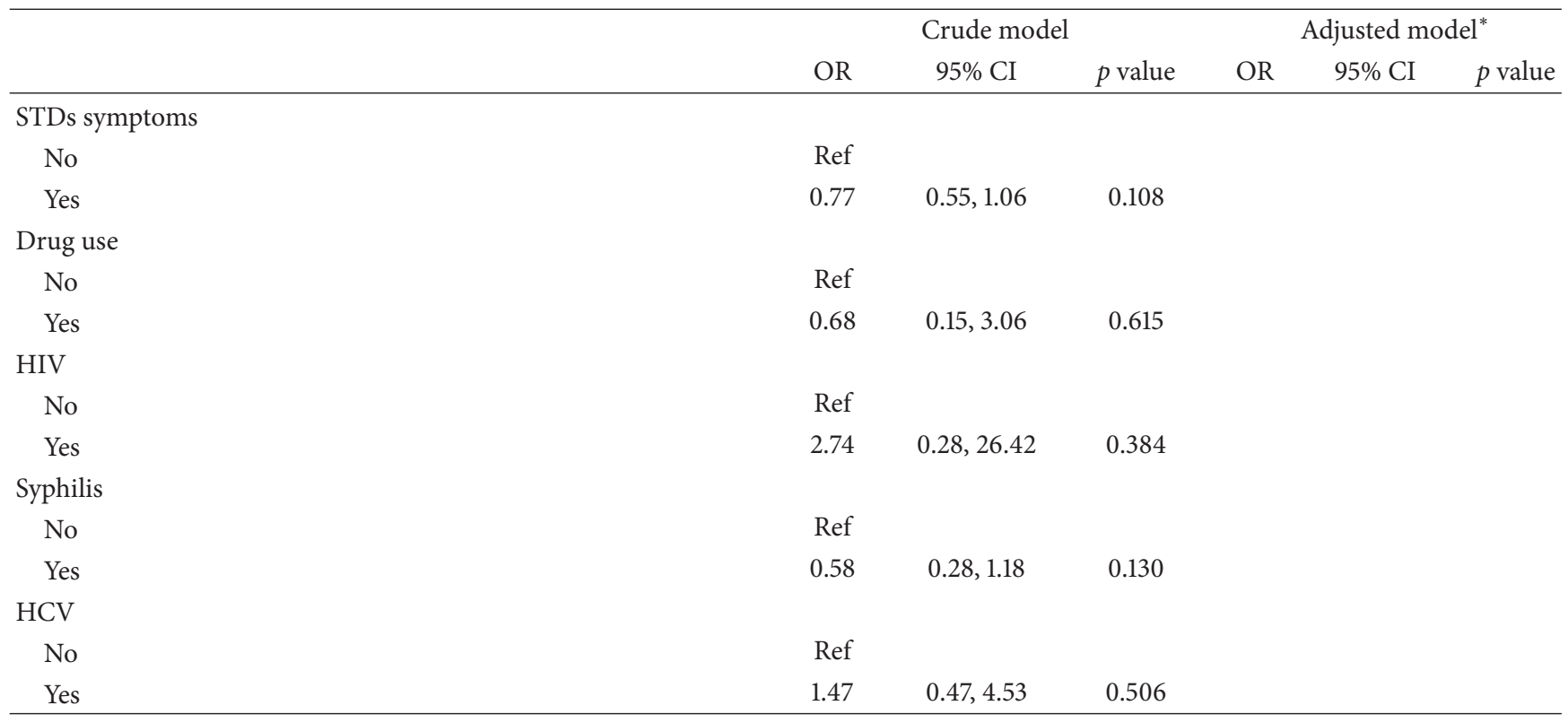

Note. ${ }^{*}$ Adjusted for age, education, marital status, and monthly income.

the literature but poorly addressed in public health programs; therefore more attention needs to be paid to the mental health wellbeing of FSWs in China [9].

Our study had several limitations. Due to the nature of convenience sampling, our sample may not be representative of FSWs in other parts of China. As the data were collected through face-to-face interviews, our study may be subject to social desirability bias, which might have led to exposure or confounder misclassifications. Even though we adjusted for age, education, marital status, and monthly income in the multivariate analysis models, our study might still have residual confounding due to the remaining unknown or unadjusted confounders. We acknowledge the potential differences in various depression assessment scales. When comparing proportion of depression among different studies, the difference we detect may vary from the true difference if a universal scale was used in those studies.

In conclusion, Chinese FSWs were suffering from extremely high level of depression. Results of our study indicated prevalent depression was relatively high among Chinese FSWs, which may contribute to the increase in HIV/STD transmission among FSWs and their clients/sexual partners. Future intervention should strengthen symptom management after education promotion, involve primary sex partners of FSWs, and integrate mental health service in comprehensive intervention to prevent depression among Chinese FSWs.

\section{Competing Interests}

The authors declare that there are no competing interests.

\section{Authors' Contributions}

Hongcheng Shen drafted this manuscript. Heping Zheng was responsible for the study design. Huachun Zou contributed to the revising and finalization of the paper. Weiming Tang and Bin Yang contributed to the conceptualization and finalization of the paper. Shujie Huang took part in the study design and was in charge of the coordination of the field work. Fengying Liu, Lei Chen, and Ye Zhang were in charge of data collection and quality control of the study. Xiaomin Luo managed the literature searches and summaries of previous related work. Peizhen Zhao analyzed the data. Hongcheng Shen and Huachun Zou contributed equally to this manuscript.

\section{Acknowledgments}

The authors would like to thank all local doctors from hospitals affiliated to the Chronic Diseases Prevention and Control Centers in Zhuhai, Nanshan, and Yingde. They extend thanks to all participants in the study.

\section{References}

[1] S. Baral, C. Beyrer, K. Muessig et al., "Burden of HIV among female sex workers in low-income and middle-income countries: a systematic review and meta-analysis," The Lancet Infectious Diseases, vol. 12, no. 7, pp. 538-549, 2012.

[2] Y. Huang, G. E. Henderson, S. Pan, and M. S. Cohen, "HIV/AIDS risk among brothel-based female sex workers in China: assessing the terms, content, and knowledge of sex work," Sexually Transmitted Diseases, vol. 31, no. 11, pp. 695-700, 2004. 
[3] M. Lau, China's Sex Industry Flourishing Despite Dangerous Conditions and Corrupt Police, South China Morning Post, 2014, http://www.scmp.com/news/china/article/1439543/chinas-sexindustry-flourishing-despite-dangerous-conditions-and-corrupt.

[4] W. L. Parish, E. O. Laumann, M. S. Cohen et al., "Populationbased study of chlamydial infection in China: a hidden epidemic," Journal of the American Medical Association, vol. 289, no. 10, pp. 1265-1273, 2003.

[5] L. Wang, N. Wang, L. Wang et al., "The 2007 estimates for people at risk for and living with HIV in China: progress and challenges," Journal of Acquired Immune Deficiency Syndromes, vol. 50, no. 4, pp. 414-418, 2009.

[6] China Ministry of Health UW, National Center for AIDS/STD Control and Prevention, China CDC, Report of epidemic situation of AIDS in China, 2011.

[7] S. Su, E. P. F. Chow, K. E. Muessig et al., "Sustained high prevalence of viral hepatitis and sexually transmissible infections among female sex workers in China: a systematic review and meta-analysis," BMC Infectious Diseases, vol. 16, no. 1, article no. 2, 2016.

[8] Y. Hong, X. Li, X. Fang, and R. Zhao, "Depressive symptoms and condom use with clients among female sex workers in China," Sexual Health, vol. 4, no. 2, pp. 99-104, 2007.

[9] C. Zhang, X. Li, Y. Hong, S. Su, and Y. Zhou, "Relationship between female sex workers and gatekeeper: the impact on female sex worker's mental health in China," Psychology, Health \& Medicine, vol. 19, no. 6, pp. 656-666, 2014

[10] C. Zhang, Y. Hong, X. Li, S. Qiao, Y. Zhou, and S. Su, "Psychological stressors in the context of commercial sex among female sex workers in China," Health Care for Women International, vol. 36, no. 7, pp. 753-767, 2015.

[11] A. Roxburgh, L. Degenhardt, and J. Copeland, "Posttraumatic stress disorder among female street-based sex workers in the greater Sydney area, Australia," BMC Psychiatry, vol. 6, article no. $24,2006$.

[12] J. A. Sherwood, A. Grosso, M. R. Decker et al., "Sexual violence against female sex workers in the Gambia: a cross-sectional examination of the associations between victimization and reproductive, sexual and mental health," BMC Public Health, vol. 15, no. 1, article no. 270, 2015.

[13] R. A. Sagtani, S. Bhattarai, B. R. Adhikari, D. Baral, D. K. Yadav, and P. K. Pokharel, "Violence, HIV risk behaviour and depression among female sex workers of eastern Nepal," BMJ Open, vol. 3, no. 6, 2013.

[14] J. Gu, J. T. F. Lau, M. Li et al., "Socio-ecological factors associated with depression, suicidal ideation and suicidal attempt among female injection drug users who are sex workers in China," Drug and Alcohol Dependence, vol. 144, pp. 102-110, 2014.

[15] Y. Hong, C. Zhang, X. Li, W. Liu, and Y. Zhou, "Partner violence and psychosocial distress among female sex workers in China," PLoS ONE, vol. 8, no. 4, Article ID e62290, 2013.

[16] J. T. F. Lau, H. Y. Tsui, S. P. Y. Ho, E. Wong, and X. Yang, "Prevalence of psychological problems and relationships with condom use and HIV prevention behaviors among Chinese female sex workers in Hong Kong," AIDS Care, vol. 22, no. 6, pp. 659-668, 2010.

[17] D. Goldberg, The Detection of Psychiatric Illness by Questionnaire: A Technique for the Identification and Assessment of NonPsychotic Psychiatric Illness, Oxford University Press, London, UK, 1972.

[18] X. Zhou, H. Song, M. Hu et al., "Risk factors of severity of post-traumatic stress disorder among survivors with physical disabilities one year after the Wenchuan earthquake," Psychiatry Research, vol. 228, no. 3, pp. 468-474, 2015.

[19] S. N. Zulkefly and R. Baharudin, "Using the 12-item General Health Questionnaire (GHQ-12) to assess the psychological health of Malaysian college students," Global Journal of Health Science, vol. 2, no. 1, pp. 73-80, 2010.

[20] S. Su, X. Li, L. Zhang, D. Lin, C. Zhang, and Y. Zhou, "Age group differences in HIV risk and mental health problems among female sex workers in Southwest China," AIDS Care Psychological and Socio-Medical Aspects of AIDS/HIV, vol. 26, no. 8, pp. 1019-1026, 2014.

[21] M. D. Ulibarri, S. Roesch, M. G. Rangel, H. Staines, H. Amaro, and S. A. Strathdee, “'Amar te Duele' ('love hurts'): sexual relationship power, intimate partner violence, depression symptoms and HIV risk among female sex workers who use drugs and their non-commercial, steady partners in Mexico," AIDS and Behavior, vol. 19, no. 1, pp. 9-18, 2015.

[22] L. Murray, L. Moreno, S. Rosario, J. Ellen, M. Sweat, and D. Kerrigan, "The role of relationship intimacy in consistent condom use among female sex workers and their regular paying partners in the Dominican Republic," AIDS and Behavior, vol. 11, no. 3, pp. 463-470, 2007.

[23] M. D. Ulibarri, S. A. Strathdee, R. Lozada et al., "Condom use among female sex workers and their non-commercial partners: effects of a sexual risk intervention in two Mexican cities," International Journal of STD \& AIDS, vol. 23, no. 4, pp. 229234, 2012.

[24] C. Wang, S. E. Hawes, A. Gaye et al., "HIV prevalence, previous HIV testing, and condom use with clients and regular partners among Senegalese commercial sex workers," Sexually Transmitted Infections, vol. 83, no. 7, pp. 534-540, 2007. 


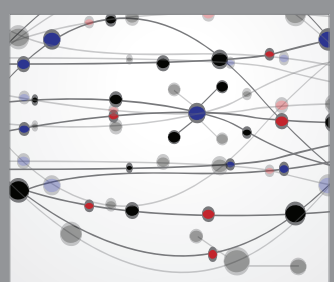

The Scientific World Journal
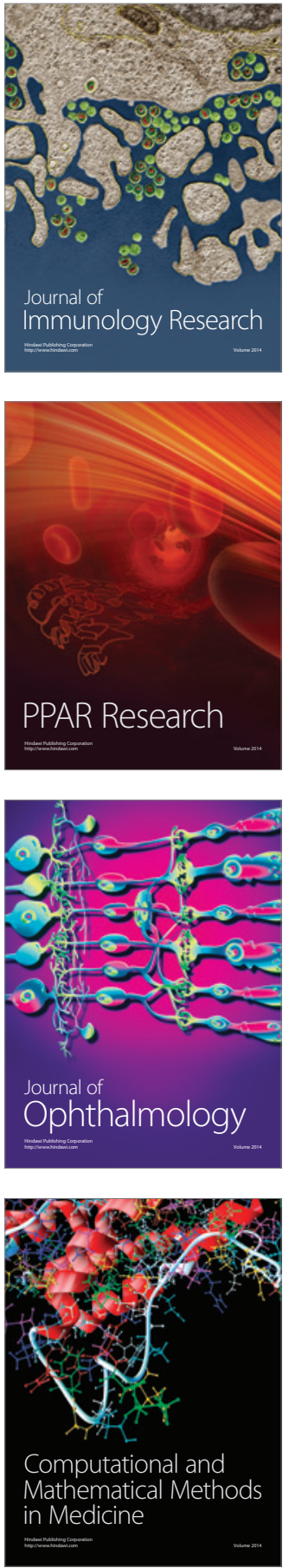

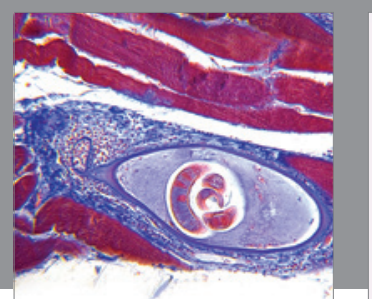

Gastroenterology Research and Practice

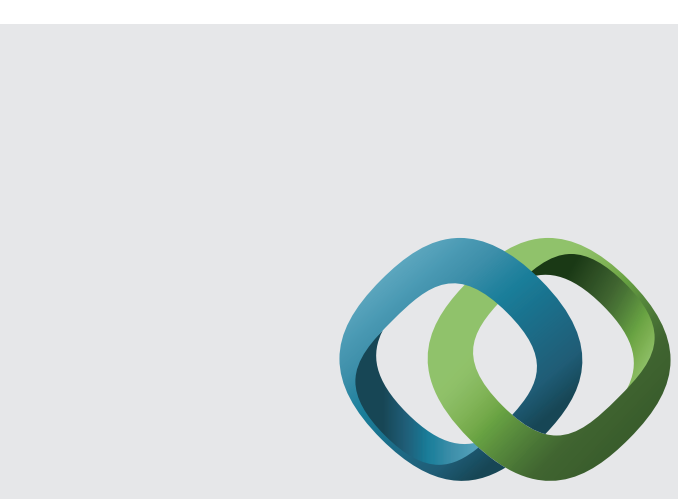

\section{Hindawi}

Submit your manuscripts at

http://www.hindawi.com
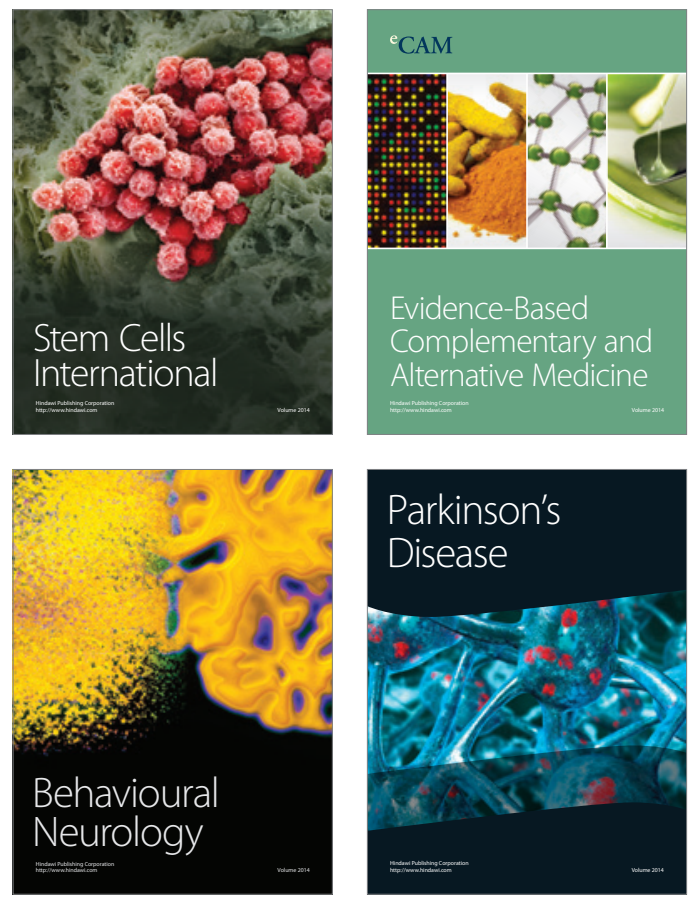
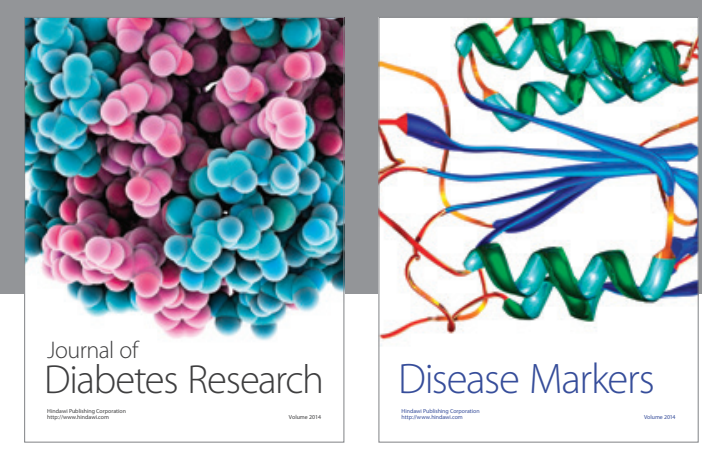

Disease Markers
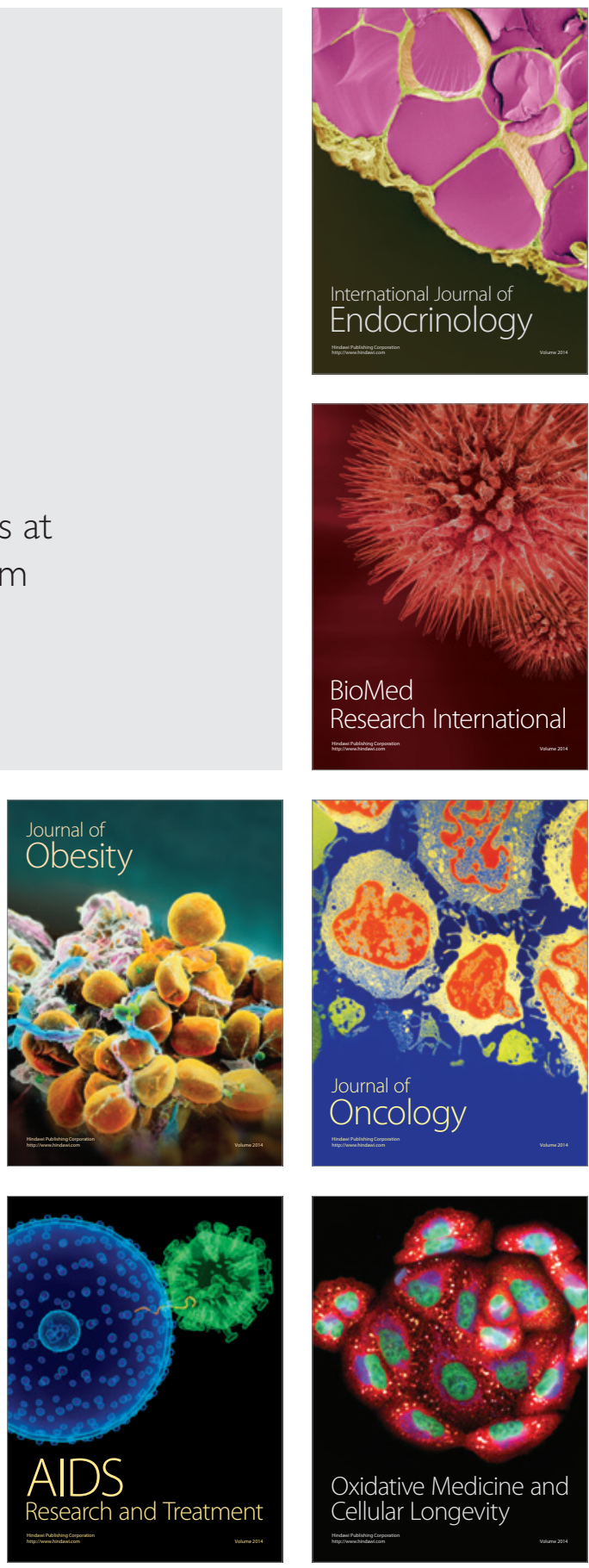